\title{
VARIABILIDADE ESPAÇO-TEMPORAL DAS VARIÁVEIS BIOFÍSICAS NO SEMIÁRIDO DO RIO GRANDE DO NORTE, BRASIL
}

\author{
Hélio Nogueira Bezerra ${ }^{1}$ \\ Joel Medeiros Bezerra ${ }^{2}$ \\ Caio Sérgio Pereira de Araújo ${ }^{3}$ \\ Arthur Mattos ${ }^{4}$
}

Resumo: A determinação de parâmetros biofísicos, tais como o Índice de Vegetação por Diferença Normalizada (NDVI) e a Temperatura de superfície (Ts), são de extrema importância para estudos de mudanças climáticas nos ambientes em geral. Com isso, o objetivo do presente estudo foi analisar a variabilidade espaço-temporal das variáveis biofísicas de NDVI e Ts, utilizando técnicas e produtos de sensoriamento remoto, em área heterogênea, com predominância de cobertura vegetal de Caatinga, associando as respostas das variações a eventos sazonais de precipitação e temperatura média diária do ar. Os resultados obtidos evidenciam o comportamento da vegetação de Caatinga mediante a distribuição temporal da pluviosidade, apresentando resultados de NDVI maiores para os períodos com maior disponibilidade hídrica e de Ts menor, devido à influência do desenvolvimento de biomassa verde sobre essas duas variáveis.

Palavras-chave: Algoritmo. Caatinga. Sensoriamento Remoto. Variáveis biofísicas.

\section{SPATIO-TEMPORAL VARIABILITY OF THE BIOPHYSICAL VARIABLES IN THE SEMI-ARID REGION OF RIO GRANDE DO NORTE, BRAZIL}

Abstract: The determination of biophysical parameters, such as the Normalized Difference Vegetation Index (NDVI) and the surface temperature (Ts), are of extreme importance for studies of climate changes in the environment in general. With this, the aim of the present study was to analyze the spatio-temporal variability of the biophysical variables of Ts and NDVI, using remote sensing techniques and products, in heterogeneous area, with predominance of Caatinga vegetal cover, associating the responses of the seasonal events of precipitation $(\mathrm{P})$ and average daily air temperature (Tar). The results obtained showed clearly the behavior of Caatinga vegetation upon the temporal distribution of rainfall, presenting results of NDVI higher for the periods with higher water availability and of Ts smaller, due to influence of green biomass development on these two variables.

Keywords: Algorithm. Caatinga. Remote sensing. Biophysics variables.

\footnotetext{
1 Universidade Federal do Rio Grande do Norte - UFRN, Campus Universitário Lagoa Nova, NatalRN, Brasil, helionogueirab@gmail.com, https://orcid.org/0000-0001-5939-2043

2 Universidade Federal Rural do Semi-Árido - UFERSA, Departamento de Engenharias e Tecnologia DETEC, Pau dos Ferros-RN, Brasil, joel.medeiros@ufersa.edu.br, https://orcid.org/0000-0002-81504125

3 Universidade Federal Rural do Semi-Árido - UFERSA, Departamento de Engenharias e Tecnologia DETEC, Pau dos Ferros-RN, Brasil, caiosergio18@hotmail.com, https://orcid.org/0000-0002-67477028

${ }^{4}$ Universidade Federal do Rio Grande do Norte - UFRN, Campus Universitário Lagoa Nova, NatalRN, Brasil, armattos@ct.ufrn.br. In memoria
} 


\section{VARIABILIDAD ESPACIO-TEMPORAL DE LAS VARIABLES BIOFÍSICAS EN EL SEMIARIO DEL RÍO GRANDE DEL NORTE, BRASIL}

Resumen: La determinación de parámetros biofísicos, tales como el Índice de Vegetación por Diferencia Normalizada (NDVI) y la Temperatura de superficie (Ts), son de extrema importancia para estudios de cambio climático en los ambientes en general. Con ello, el objetivo del presente estudio fue analizar la variabilidad espaciotemporal de las variables biofísicas de NDVI y Ts, utilizando técnicas y productos de sensoriamiento remoto, en área heterogénea, con predominancia de cobertura vegetal de Caatinga, asociando las respuestas de las variaciones a eventos estacional de precipitación y temperatura media diaria del aire. Los resultados obtenidos evidencian el comportamiento de la vegetación de Caatinga mediante la distribución temporal de la pluviosidad, presentando resultados de NDVI mayores para los períodos con mayor disponibilidad hídrica y de Ts menor, debido a la influencia del desarrollo de biomasa verde sobre esas dos variables.

Palabras clave: Algoritmo. Caatinga. Detección remota. Variables biofísicas.

\section{Introdução}

O índice de vegetação por diferença normalizada (NDVI) tem uma relação direta com o vigor da vegetação, sendo assim, é possível o mapeamento de áreas com diferentes índices de cobertura vegetal e de biomassa (SILVA, 2009). Este índice tem sido amplamente utilizado em várias aplicações operacionais, incluindo mapeamentos de classificação de cobertura da terra (FECHINE; GALVÍNCIO, 2008), detecção de mudanças e monitoramento ambiental (ARAÚJO et al., 2011), e estudos de evapotranspiração (ARRAES et al., 2012), que incluem o conhecimento do balanço de energia e da temperatura da superfície (Ts), elementos que influenciam diretamente os fenômenos ligados à vegetação e suas condições.

O NDVI e a Ts constituem-se em parâmetros notadamente influenciados por variações no clima em escala local, além das ações antrópicas, cujas estimativas são de grande utilidade em trabalhos de gestão dos recursos hídricos e monitoramento ambiental.

Segundo Cunha et al. (2012), com o monitoramento dos parâmetros biofísicos é possível diagnosticar alterações antrópicas e naturais na paisagem, e orientar o estabelecimento de políticas de reversão de um quadro de degradação ambiental, o que torna esses parâmetros úteis no estabelecimento de uso e ocupação do solo de forma sustentável. 
Apesar de ser o único bioma natural brasileiro inteiramente restrito ao território nacional, pouca atenção tem sido dada à conservação da diversificada e marcante paisagem de Caatinga. Outro aspecto relevante é que, o mesmo considerando a contribuição de sua biota, a grande biodiversidade tem sido, ainda assim, subestimada (BEZERRA et al., 2014). De acordo com Garda (1996), somente a presença da vegetação de Caatinga adaptada às condições locais, tem impedido a transformação do Nordeste brasileiro num imenso deserto.

Em virtude da importância da dinâmica espaço-temporal da Caatinga em regiões semiáridas do Nordeste brasileiro, pesquisas são necessárias para compreender o comportamento dos parâmetros biofísicos, face às mudanças climáticas e ações antropogênicas.

Para Oliveira et al. (2012) a quantificação de parâmetros biofísicos de temperatura da superfície e alguns índices de vegetação, por meio de instrumentos convencionais, em escala regional, representaria um grande dispêndio de recursos materiais e humanos. Portanto, recomendam o uso do sensoriamento remoto orbital, que se mostra como uma opção economicamente mais viável em grandes áreas.

Corroborando com tal pressuposto, Silva et al. (2010) identificaram alterações climáticas decorrentes de mudanças no uso da terra mediante aplicação dos produtos de sensoriamento remoto para estimativa dos parâmetros biofísicos, nas Bacias do Rio Mogi-Guaçu, SP, e do Baixo Jaguaribe, CE, e verificaram boa concordância entre as imagens obtidas e as medições micro meteorológicas existentes em campo nas áreas do estudo.

Diante do exposto, o objetivo do presente estudo foi analisar a variabilidade espaço-temporal das variáveis biofísicas de NDVI e Ts, em área heterogênea, com predominância de cobertura vegetal de Caatinga, utilizando técnicas e produtos de sensoriamento remoto, e associando as respostas das variações a eventos sazonais de precipitação $(\mathrm{P})$ e temperatura média diária do ar (Tar), medidos em estação meteorológica convencional.

\section{Material e Métodos}


A área de estudo compreende o entorno do reservatório Dourado (coordenada geográfica do centroide: $06^{\circ} 14^{\prime} 25^{\prime \prime} \mathrm{S} ; 36^{\circ} 30^{\prime} 15^{\prime \prime} \mathrm{O}$ ), que está situado no município de Currais Novos, região semiárida do estado do Rio Grande do Norte.

O reservatório Dourado é fonte de alimentos, através da pesca, e do abastecimento público de água da cidade, além de ser explorado para agricultura irrigada e dessedentação de animais de pequenas propriedades rurais situadas às margens do reservatório. A sua capacidade máxima de acumulação está em torno de $10.321 .600,00 \mathrm{~m}^{3}$ de água, com uma área superficial de $3,16 \mathrm{~km}^{2}$ e profundidade máxima de $10 \mathrm{~m}$.

Sendo que a área de estudo compreende cerca de $2,3 \mathrm{~km}^{2}$ do entorno do reservatório. Essa área é caracterizada pela heterogeneidade na cobertura e uso do solo, que vai desde vegetação nativa até pequenos lotes de pecuária e agricultura.

A área de estudo está inserida em região semiárida, com precipitação média anual de $476 \mathrm{~mm}$, e com predominância da vegetação Caatinga subdesértica do Seridó, a mais seca do estado, com arbustos e árvores ralas e de xerofitismo mais acentuado. Esse cenário é formado por árvores espalhadas em uma matriz arbustiva, que geralmente é formada pela jurema preta devido ao seu caráter pioneiro. As principais espécies encontradas na região são: pereiro (Aspidosperma pyrifolium), faveleiro (Cnidoscolus phyllacanthus), facheiro (Pilosocereus glaucescens), macambira (Bromelia laciniosa), mandacaru (Cereus jamacaru), xique-xique (Pilosocereus gounellei) e jurema-preta (Mimosa tenuiflora) (RIO GRANDE DO NORTE, 2008).

O clima do Seridó é seco, com altas taxas de evapotranspiração que contribuem para a formação de rios temporários e pouca retenção de água no solo. Isto dificulta a produção agrícola em geral e a vida da população local. Dessa maneira, torna-se necessária a construção de adutoras e barragens que amenizem a situação (BEZERRA JÚNIOR; SILVA, 2007).

A partir da Figura 1 pode-se observar a distribuição espacial das principais atividades e/ou tipos de uso e ocupação das áreas do entorno do reservatório. 


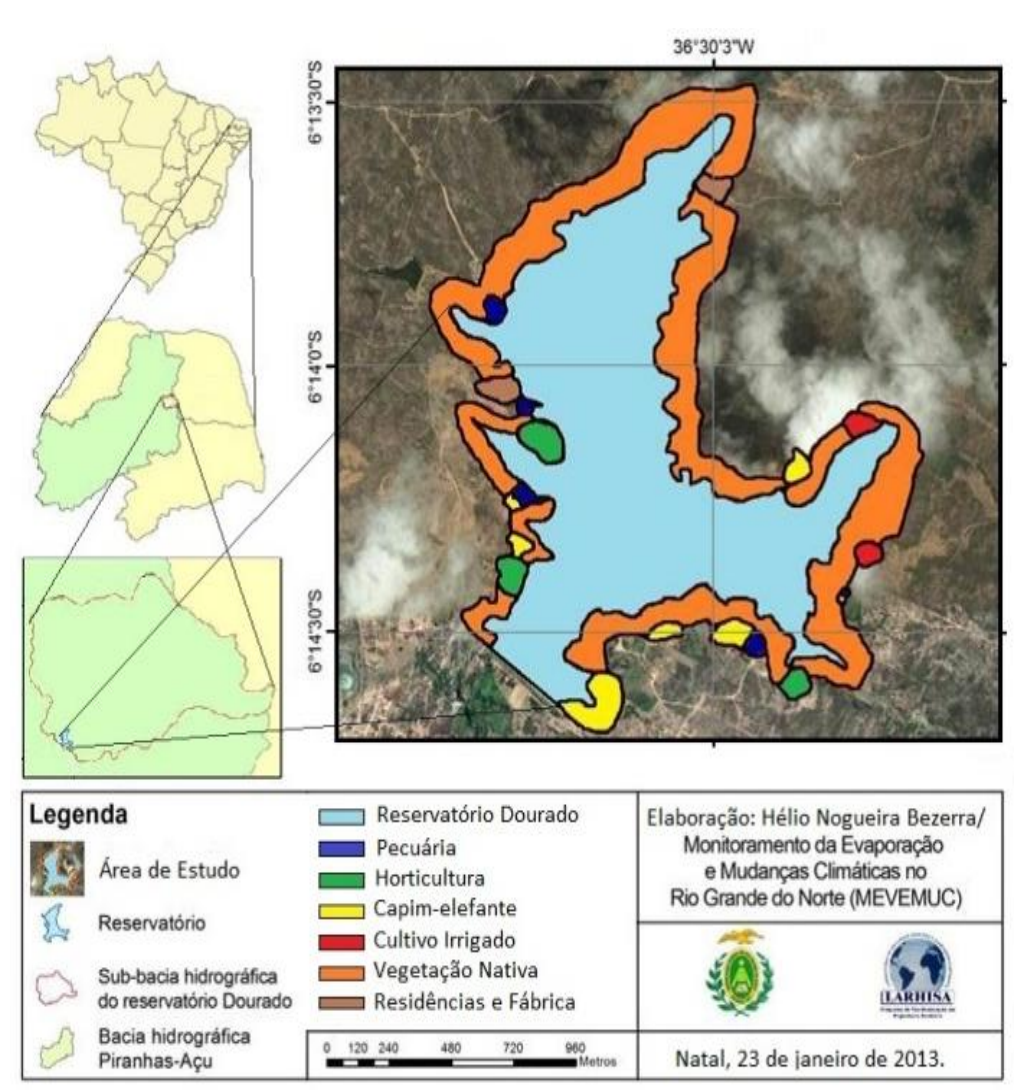

Figura 1: Mapa de localização da área de estudo com os principais tipos de coberturas e usos do solo no entorno do reservatório Dourado.

Segundo o Plano Nacional de Combate à Desertificação (PNCD), define desertificação como a degradação da terra nas zonas áridas, semiáridas e sub-úmidas secas, resultantes de fatores diversos tais como as variações climáticas e as atividades antrópicas. Currais Novos está inserido em área susceptível à desertificação em categoria "muito grave" (RIO GRANDE DO NORTE, 2008). Tal situação torna imprescindível a realização de estudos de monitoramento e gestão dos recursos naturais nesse município, a fim de evitar danos, principalmente ao reservatório Dourado, fonte de abastecimento de água e renda na cidade.

No entanto, o processo de degradação que a vegetação seridoense vem sofrendo, destacado por Bezerra Júnior e Silva (2007), revela que mesmo tendo uma predominância de árvores e arbustos baixos, é possível encontrar indícios de que havia uma quantidade maior de árvores altas, em virtude da diversidade de espécies, associado ao estado de resiliência da atual cobertura vegetal. Mesmo assim, como a 
vegetação é diretamente influenciada pelo solo e clima, que varia sazonal e espacialmente, é possível encontrar pontos de predominância arbustiva e outros exclusivamente arbóreos.

Nesse contexto, o solo é raso e pouco desenvolvido, marcado por afloramentos rochosos e aparecimento de pedregulhos na superfície, caracterizando o neossolo litólico. Esse tipo de solo apresenta drenagem moderada, denotando que a água absorvida é perdida lentamente, porém mantém o solo úmido por um curto período de tempo (EMBRAPA, 1971).

Os valores das variáveis de superfície (temperatura do ar, velocidade do vento, insolação, precipitação e umidade relativa do ar) medidas em campo foram obtidos em estação meteorológica convencional do Instituto Nacional de Meteorologia (INMET), localizada na fazenda da Empresa de Pesquisa Agropecuária do Rio Grande do Norte (EMPARN), situada no município de Cruzeta/RN, que fica a menos de $40 \mathrm{~km}$ da área de estudo, por ser a única estação operante nas proximidades da área em estudo.

Utilizou-se das variáveis meteorológicas de precipitação pluviométrica diária e temperatura média diária do ar, a fim de avaliar a sazonalidade temporal. Os dados foram coletados de 01 de janeiro de 2005 a 31 de dezembro de 2010, abrangendo o período das imagens selecionadas para a aplicação do modelo.

Para o cálculo do NDVI e da Ts foram utilizadas imagens geradas pelo sensor TM (Thematic Mapper) do satélite Landsat-5, com cobertura da órbita/ponto 215/64. $\mathrm{Na}$ Tabela 1 verifica-se a lista das imagens TM/Landsat-5 selecionadas para 0 presente estudo, assim como sua respectiva hora de imageamento, dia juliano ou dia sequencial do ano, distância relativa inversa Terra-Sol (dr) e ângulo zenital de elevação solar $(Z)$.

Tabela 1: Dados das imagens do satélite Landsat-5/ sensor Thematic Mapper (TM).

\begin{tabular}{cccccc}
\hline Data da imagem & $\mathbf{1 3 / 0 7 / 2 0 0 5}$ & $\mathbf{1 9 / 0 7 / 2 0 0 7}$ & $\mathbf{1 9 / 0 6 / 2 0 0 8}$ & $\mathbf{0 8 / 0 7 / 2 0 0 9}$ & $\mathbf{0 9 / 0 6 / 2 0 1 0}$ \\
\hline Hora $\left(\mathrm{GMT}^{\star}\right)$ & $12: 22: 35$ & $12: 28: 27$ & $12: 22: 24$ & $12: 23: 34$ & $12: 25: 30$ \\
Dia Sequencial & 194 & 200 & 171 & 189 & 160
\end{tabular}




\begin{tabular}{cccccc}
$\mathrm{dr}$ & 0,968 & 0,968 & 0,968 & 0,967 & 0,969 \\
$\mathrm{Z}$ (Graus) & 48,3959 & 49,9648 & 48,1670 & 48,2933 & 49,4188 \\
\hline
\end{tabular}

${ }^{*}$ GMT: Greenwich Mean Time

O processamento das imagens foi executado com o software ERDAS Imagine 9.1, a partir da ferramenta Model Maker, que permite a implementação de algoritmos, a partir de modelos alfanuméricos, possibilitando a modelagem dos sistemas matriciais de imagens raster, produzindo mapas com a distribuição espacial das variáveis desejadas.

Antes do processo de tratamento digital de imagens propriamente dito, foi realizado um pré-processamento, que consistiu no empilhamento das sete bandas que compõem as imagens selecionadas. Depois, as imagens empilhadas foram registradas a partir de imagem ortorretificada disponibilizada no site da NASA (USGS), em seguida recortadas de acordo com os limites da área de interesse do estudo.

Após o pré-processamento das imagens, foi realizada a calibração radiométrica, que gerou a radiância espectral a partir da conversão do número digital (ND) de cada pixel da imagem. Os valores das radiâncias espectrais mínima (a) e máxima (b) utilizadas na calibração radiométrica foram retirados da Tabela 2, proposta por Chander e Markham (2003).

Tabela 2: Descrição das bandas do sensor TM do Landsat-5, com os intervalos correspondentes de comprimento de onda, coeficientes de calibração (radiância mínima - a e máxima - b) e irradiâncias espectrais no topo da atmosfera.

\begin{tabular}{ccccc}
\hline Bandas & $\begin{array}{c}\text { Comprimento } \\
\text { de Onda }\end{array}$ & \multicolumn{2}{c}{$\begin{array}{c}\text { Coeficientes de } \\
\text { Calibração } \\
\left(\mathbf{W m}^{-2} \mathbf{s r}^{-1} \boldsymbol{\mu m}^{-1}\right)\end{array}$} & $\begin{array}{c}\text { Irradiância Espectral } \\
\text { no Topo da Atmosfera }\end{array}$ \\
\hline 1 (Azul) & $0,45-0,52$ & $-1,52$ & 193,0 & $\left(\mathbf{W m}^{-2} \boldsymbol{\mu m}^{-1}\right)$ \\
2 (Verde) & $0,52-0,60$ & $-2,84$ & 365,0 & 1957 \\
3 (Vermelho) & $0,63-0,69$ & $-1,17$ & 264,0 & 1826 \\
\end{tabular}




\begin{tabular}{|c|c|c|c|c|}
\hline $\begin{array}{c}4 \text { (IV - } \\
\text { próximo) }\end{array}$ & $0,76-0,90$ & $-1,51$ & 221,0 & 1036 \\
\hline 5 (IV - médio) & $1,55-1,75$ & $-0,37$ & 30,2 & 215 \\
\hline 6 (IV-termal) & $10,4-12,5$ & 1,2378 & 15,303 & - \\
\hline 7 (IV - médio) & $2,08-2,35$ & $-0,15$ & 16,5 & 80,67 \\
\hline
\end{tabular}

De posse das cartas temáticas de radiância espectral de cada banda, informações sobre o cosseno do ângulo zenital do Sol ( $\cos Z)$ e do fluxo direcional espectral no topo da atmosfera ( $\mathrm{K} \lambda \mathrm{i})$ de cada banda, estimou-se a refletância planetária de cada banda, seguindo com os procedimentos detalhados na Tabela 3 até a estimativa das variáveis biofísicas (NDVI, SAVI, IAF e temperatura de superfície).

Tabela 3: Etapas do processamento das variáveis biofísicas pelo algoritmo SEBAL.

\section{Etapa 1 - Calibração Radiométrica}

$$
\mathrm{L}_{\lambda \mathrm{i}}=\mathrm{a}_{\mathrm{i}}+\left(\frac{\mathrm{b}_{\mathrm{i}}-\mathrm{a}_{\mathrm{i}}}{255}\right) \mathrm{ND}
$$

a e b são as radiâncias espectrais mínimas e máximas $\left(\mathrm{Wm}^{-2} \mathrm{Sr}^{-1} \mu \mathrm{m}^{-1}\right)$; ND é a intensidade do pixel (número digital - número inteiro de 0 a 255); i são as bandas (1, 2, 3, 4, 5, 6 e 7) do sensor TM / Landsat 5.

\section{Etapa 2 - Reflectância Planetária}

$L \lambda i$ é a radiância espectral de cada banda; $k \lambda i$ é a irradiância solar espectral no topo da atmosfera $\left(\mathrm{Wm}^{-}\right.$

$$
\rho_{\lambda \mathrm{i}}=\frac{\pi \cdot \mathrm{L}_{\lambda \mathrm{i}}}{\mathrm{k}_{\lambda \mathrm{i}} \cdot \cos Z \cdot \mathrm{d}_{\mathrm{r}}}
$$
$\left.{ }^{2} \mu \mathrm{m}^{-1}\right)$; $Z$ é o ângulo zenital solar e $d r$ a razão entre a distância média Terra-Sol dada por $d=1+0,033 \cdot \cos (D S A \cdot 2 \pi / 365)$, onde DSA é o Dia Sequencial do ano.

\section{Etapa 3 - Índices de Vegetação}

$$
\mathrm{NDVI}=\frac{\rho_{\mathrm{IV}}-\rho_{\mathrm{V}}}{\rho_{\mathrm{IV}}+\rho_{\mathrm{V}}}
$$

$\operatorname{SAVI}=\frac{(1+\mathrm{L})\left(\rho_{\mathrm{IV}}-\rho_{\mathrm{V}}\right)}{\left(\mathrm{L}+\rho_{\mathrm{IV}}+\rho_{\mathrm{V}}\right)}$

$\mathrm{IAF}=-\frac{\ln \left(\frac{0,69-\mathrm{SAVI}}{0,59}\right)}{0,91}$
$\rho_{I V}, \rho_{V}$ correspondem, respectivamente, as bandas 4 e 3 do sensor TM / Landsat 5.

$L$ é uma variável de valor 0,25 (usado para vegetação densa), 0,5 (usado para vegetação intermediária) e 1,0 (usado para pouca vegetação). O valor usado no estudo para essa variável foi 1,0. 


\section{Etapa 4 - Emissividade}

$\varepsilon_{\mathrm{NB}}=0,97+0,0033 . \mathrm{IAF}$

Para pixels com IAF $\geq 3, \varepsilon N B=\varepsilon_{0}=0,98$ e para corpos

$\varepsilon_{0}=0,95+0,01 . \mathrm{IAF}$ de água (NDVI < 0) $\varepsilon_{N B}=0,99$ e $\varepsilon_{0}=0,985$, conforme recomendações de Allen et al. (2002).

\section{Etapa 5 - Temperatura da Superfície}

$\mathrm{T}_{\mathrm{s}}=\frac{\mathrm{K}_{2}}{\ln \left(\frac{\varepsilon_{\mathrm{NB}} \mathrm{K}_{1}}{\mathrm{~L}_{\lambda, 6}}+1\right)}$

$\mathrm{K} 1=607,76 \mathrm{Wm}^{-2} \mathrm{sr}^{-1} \mu^{-1}$ e K2 $=1260,56 \mathrm{Wm}^{-2} \mathrm{sr}^{-1} \mu \mathrm{m}^{-}$

1 são as constantes de calibração da banda termal do sensor TM / Landsat 5. A radiância espectral da banda termal $L_{\lambda 6}$ e a emissividade $\varepsilon_{N B}$.

Após a implementação dos procedimentos do algoritmo SEBAL e a elaboração dos mapas das variáveis biofísicas (NDVI e Ts), os valores de cada pixel foram exportados para planilhas do EXCEL para realizar a estatística descritiva, a fim de detectar as características tendenciais de posição e de dispersão dos dados obtidos.

\section{Resultados e Discussão}

A aplicação do algoritmo para a obtenção das variáveis biofísicas a partir de produtos orbitais de sensoriamento remoto possibilitou boa representação de sua variabilidade espacial, permitindo a demonstração das respostas espectrais dos diversos usos do solo, como pontos de acúmulo de água (reservatório), solo exposto e vegetação densa ou rala, juntamente com o conhecimento do local a partir do mapa de uso e ocupação do solo, imprescindível para essa avaliação.

Como as imagens selecionadas se apresentaram em sequência cronológica (ver Figura 2), foi possível analisar também a variabilidade temporal, sendo viável a aplicação desse método para a avaliação sazonal das regiões de alta heterogeneidade de uso e ocupação do solo e de clima semiárido, com grande variação na disponibilidade hídrica, que causa a alteração da cobertura do solo. 


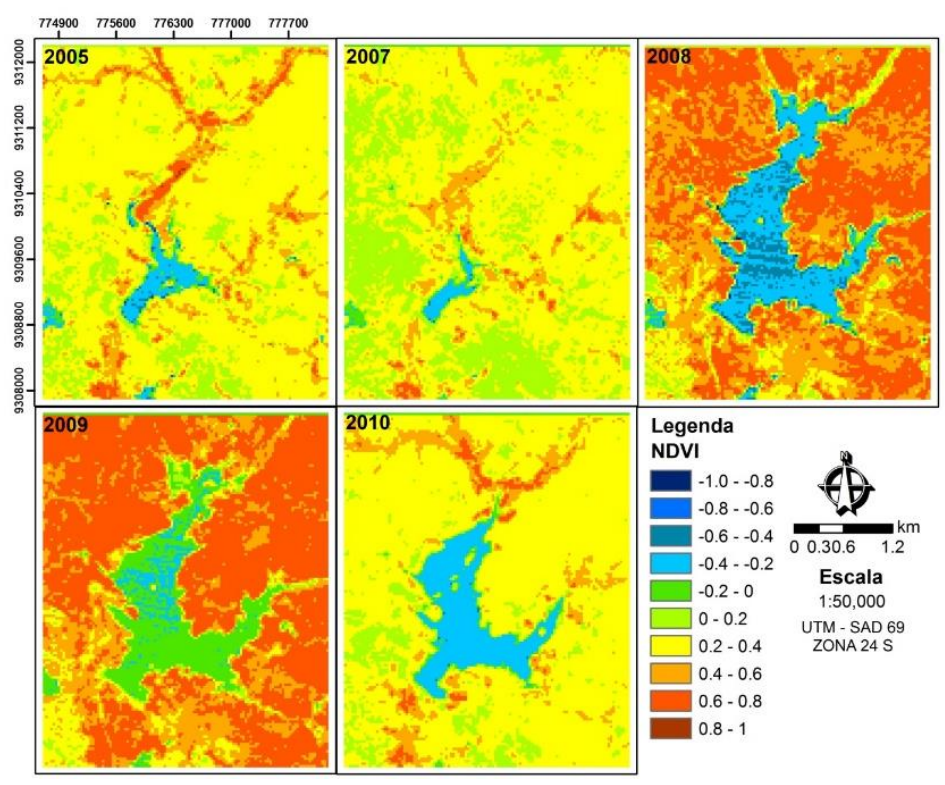

Figura 2: Distribuição espaço temporal do NDVI para a área de estudo (Currais Novos/RN).

Observam-se claramente diferenças de valores entre as imagens 2008 ou 2009 e as demais, pois as primeiras ocorreram em período com maior disponibilidade hídrica devido aos maiores volumes de precipitação, obtendo-se assim, valores maiores de NDVI, resposta da vegetação de Caatinga à presença de água disponível para o desenvolvimento de sua biomassa. Já nos demais anos, que se inseriram em período de estiagem, os valores de NDVI reduziram, causa da redução drástica nas folhagens da vegetação devido ao mecanismo fisiológico de defesa da Caatinga e a maior exposição do solo à radiação solar global.

Com relação à variação temporal, os valores obtidos de NDVI foram coerentes com a sazonalidade dos dados de precipitação e temperatura do ar medidos em estação meteorológica convencional, ratificando a aplicabilidade do método em regiões semiáridas. Nos anos de 2005 e 2007, por passar por um período de estiagem, com índices pluviométricos baixos, os valores de NDVI foram menores (entre 0 e 0,4), nos anos de 2008 e 2009, com maior disponibilidade hídrica e precipitação pluvial anual acima da média, os valores obtidos para NDVI foram maiores (entre 0,4 e 0,8), e no ano de 2010, apesar do reservatório ainda se encontrar bem abastecido, a 
precipitação anual foi muito aquém da média, e por isso apresentou baixos valores de NDVI (entre 0 e 0,4).

Nas imagens de 2005, 2007 e 2010, compreendendo períodos secos, os valores de NDVI acima de 0,4 foram referentes à mata ciliar, vegetação situada nas margens dos rios, que aproveitam as águas disponíveis para suprir suas necessidades fisiológicas, e aos cultivos irrigados (capim elefante, horta e frutíferas), que apesar do baixo volume do reservatório (2005 e 2007), os produtores não cessam a irrigação.

Nas imagens de 2008 e 2009 os valores de NDVI acima de 0,4 representaram o comportamento da vegetação de Caatinga para períodos chuvosos, quando as plantas formam suas folhas (dossel foliar) para realizar a fotossíntese mediante a absorção da radiação proveniente do sol, juntamente com a água disponível no solo.

Arraes et al. (2012), Gurgel et al. (2003) e Barbosa et al. (2006) constaram alta variabilidade do NDVI em área de vegetação nativa de Caatinga, confirmando a relação direta que essa variável tem com a disponibilidade hídrica no solo para as plantas, e certificando a variabilidade sazonal encontrada nesse estudo, assim como os valores encontrados para esse tipo de vegetação, variando de 0,25 a 0,8.

$\mathrm{Na}$ Tabela 4, verificam-se os valores de tendência central e de dispersão dos dados de NDVI, confirmando os resultados da variabilidade espacial e temporal expostas nos mapas da Figura 3, mostrando que as imagens de 2008 e 2009 apresentaram tendências centrais maiores em relação as demais, assim como os valores de variância e desvio padrão.

Tabela 4: Valores estatísticos descritivos aplicados sobre os dados exportados das imagens de NDVI.

\begin{tabular}{ccccccccc}
\hline Imagem & $\mathbf{N}$ & Média & Med. & Mín. & Máx. & Var. & DP & CV \\
\hline 2005 & 16588 & 0,278 & 0,269 & $-0,961$ & 0,813 & 0,025 & 0,159 & 57,040 \\
2007 & 16588 & 0,246 & 0,231 & $-0,500$ & 0,782 & 0,013 & 0,112 & 45,606 \\
2008 & 16588 & 0,406 & 0,558 & $-1,000$ & 0,832 & 0,126 & 0,355 & 87,414 \\
2009 & 16588 & 0,480 & 0,609 & $-0,391$ & 0,788 & 0,080 & 0,283 & 59,037
\end{tabular}


$\begin{array}{lllllllll}2010 & 16588 & 0,234 & 0,262 & -0,462 & 0,775 & 0,040 & 0,201 & 86,131\end{array}$

Nota: N é o Número de pixels; Med. é a Mediana; Mín. é o valor Mínimo; Máx. é o valor Máximo; Var. é a Variância; DP é o Desvio Padrão; e CV é o Coeficiente de Variação (\%).

No entanto apenas as imagens de 2008 apresentaram alto coeficiente de variação, mostrando que apesar da dispersão em relação a média ter sido alta a maior parte dos dados se concentram dentro do desvio. Isso se deve ao fato da vegetação ter se apresentado mais vigorosa nesse período, aumentando os valores de NDVI da vegetação de Caatinga e ampliando, consequentemente, a distribuição da frequência desses valores em outras classes de resultados.

Os momentos estatísticos possibilitaram ainda constatar que a imagem de 2010 apresentou alta concentração de dados dentro do desvio padrão sobre a média, fato que denota baixa variabilidade espacial. Enquanto, as imagens de 2005 e 2007 observaram-se baixa variância e desvio padrão sobre a média, além de um coeficiente de variação baixo, o que afirma um comportamento de homogeneidade espacial com relação aos valores de NDVI.

Assim como o NDVI, a Ts pode ser utilizada como indicador de mudanças da superfície do solo, proporcionando respostas espectrais as quais podem ser utilizadas para diversos fins, tais como: monitoramento ambiental com a identificação da evolução de áreas de solo exposto; uso agrícola, subsidiando o planejamento de áreas irrigadas; hidrologia fornecendo informações no manejo da água, e etc.

Os valores de Ts encontrados neste trabalho corroboraram com os valores de NDVI, mostrando serem duas variáveis diretamente relacionadas, já que quando houve grande presença de vegetação (NDVI próximo a 1) a temperatura da superfície diminuiu para áreas da superfície terrestre, devido a questão da absorção de calor (advindo da radiação solar) pelas plantas ser menor em relação ao solo exposto ou com afloramento rochoso. Esse comportamento, inversamente proporcional, entre as duas variáveis foi observado quando o NDVI possui valores positivos (de 0 a 1 ).

Essas duas variáveis biofísicas apresentaram comportamento diretamente proporcional quando o NDVI assumiu valores negativos (de -1 a 0 ), pois quanto menor 
o valor de NDVI maior o valor da temperatura de superfície. Esse caso ocorre para os pixels que compreendem o reservatório, que apresentaram valores menores de temperatura de superfície, devido a lâmina d'água refletir mais do que absorve a radiação proveniente do sol.

Verifica-se na Figura 3 a distribuição espacial da temperatura de superfície. Conforme os mapas temáticos, os menores valores foram observados para os anos de 2008 e 2009 (variando de 290,3 a 298 K), devido ao alto índice pluviométrico e consequentemente ao desenvolvimento da vegetação de Caatinga, que ameniza as condições térmicas da superfície terrestre. Nas imagens de 2007 e 2010 os valores de Ts foram maiores (variando de 302,1 a $310 \mathrm{~K}$ ) devido à baixa cobertura do solo e ao baixo índice pluviométrico, causado pela perda do dossel da vegetação de Caatinga como mecanismo fisiológico de defesa contra o período de seca.

$\mathrm{Na}$ imagem de 2005 os valores de Ts apresentaram uma tendência média, ou seja, apresentou resultados intermediários, com valores variando de 298,1 a $302 \mathrm{~K}$, já que nesse ano as precipitações foram próximas a média anual da região (480 mm), mostrando uma variabilidade da variável biofísica em torno da média para o referido ano. No entanto, observam-se valores menores de Ts para alguns pixels isolados que não são referentes ao reservatório Dourado, esses resultados estão associados à hidrografia da área de estudo, representando os rios afluentes do reservatório.

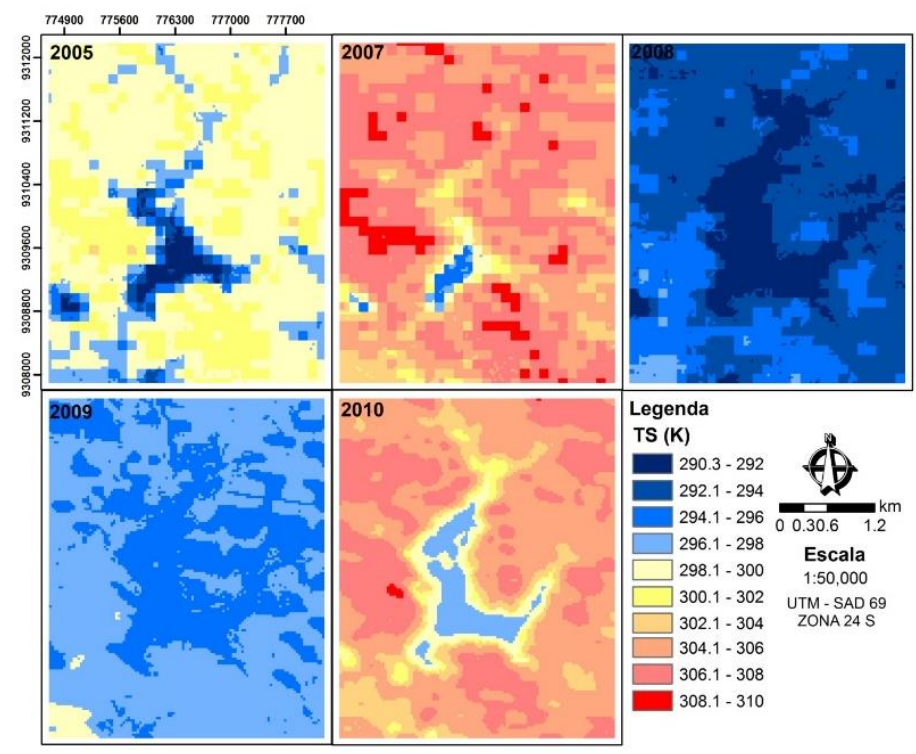


Figura 3: Variabilidade espacial e temporal da temperatura de superfície para o entorno do reservatório Dourado (Currais Novos/RN).

Podem-se constatar na Tabela 5 os valores da estatística descritiva aplicada aos valores extraídos dos pixels das imagens processadas de Ts. Os dados confirmam a tendência das imagens de 2007 e 2010 apresentarem maiores valores de Ts, possuindo desvios padrão de 2,16 e 2,57, e variância de 4,65 e 6,62, respectivamente, o que significa que os valores de Ts se dispersam em relação a média, se aproximando do máximo obtido, devido aos altos valores observados nos mapas da Figura 4, e sabendo que os baixos valores são correspondentes à lâmina de água do reservatório Dourado.

Tabela 5: Valores estatísticos descritivos aplicados sobre os dados exportados das imagens de Ts em Kelvin (K).

\begin{tabular}{ccccccccc}
\hline Imagem & $\mathbf{N}$ & Média & Med. & Mín. & Máx. & Var & DP & CV \\
\hline 2005 & 16588 & 298,85 & 299,35 & 289,70 & 302,14 & 4,37 & 2,09 & 70 \\
2007 & 16588 & 305,59 & 305,91 & 294,22 & 309,59 & 4,65 & 2,16 & 71 \\
2008 & 16588 & 293,02 & 293,02 & 290,16 & 296,95 & 1,93 & 1,39 & 47 \\
2009 & 16588 & 296,10 & 296,13 & 294,22 & 299,14 & 0,65 & 0,80 & 27 \\
2010 & 16588 & 304,49 & 305,44 & 296,86 & 308,77 & 6,62 & 2,57 & 85 \\
\hline
\end{tabular}

Nota: N é o Número de pixels; Med. é a Mediana (K); Mín. é o valor Mínimo (K); Máx. é o valor Máximo (K); Var. é a Variância; DP é o Desvio Padrão; e CV é o Coeficiente de Variação (\%).

Os valores estatísticos para o ano de 2009 mostram temperaturas baixas e uma tendência central maior devido aos baixos valores de dispersão (variância, desvio padrão e coeficiente de variação), o que é explicado pelo fato do ano apresentar pluviosidade de $1170 \mathrm{~mm}$ e o ano antecedente de $996 \mathrm{~mm}$, considerado acima da média. Desta forma, provocando um adensamento da vegetação de Caatinga e acúmulo de água no solo, que apesar de ser raso, foi contemplado com chuva de 1,2 
$\mathrm{mm}$ no período do imageamento, fato que interfere as condições atmosféricas, reduzindo a temperatura de superfície.

Outro fator que influenciou na baixa dispersão dos valores dos pixels da imagem de 2009 foi a temperatura da superfície ter baixa variação em relação à temperatura da lâmina d'água, que atingiu uma média de 22,8 ํ․

Silva et al. (2011) encontraram valores de Ts variando entre 300 e $306 \mathrm{~K}$ para áreas de solo exposto e de cobertura vegetal de Caatinga, resultados que concordaram com os valores obtidos neste estudo. Os referidos autores evidenciaram que as áreas irrigadas apresentaram Ts inferiores devido a maior disponibilidade de água no solo nessas áreas. Essa afirmativa pode ser confirmada pelas imagens 2008 e 2009, que nesse caso apresentaram maior disponibilidade de água no solo devido às precipitações nesse período.

\section{Considerações Finais}

As variáveis biofísicas, NDVI e Ts, apresentaram variabilidade temporal relacionada aos eventos sazonais de precipitação e temperatura do ar (medidos em estação meteorológica convencional), mesmo em área de entorno do reservatório.

Para a área em estudo, o comportamento espectral dos pixels das áreas heterogêneas (atividades agropecuárias) não favoreceu uma clara percepção da variabilidade espacial e temporal das variáveis biofísicas nesses pontos, por apresentarem áreas espacialmente pequenas em relação ao tamanho do pixel da imagem TM/Landsat-5.

O método utilizado para determinação das variáveis biofísicas em regiões semiáridas com grande heterogeneidade e pequenas áreas é mais eficiente com o uso de dados radiométricos de sensores de alta resolução espacial.

A aplicação do modelo apresentou-se como excelente ferramenta para o monitoramento da disponibilidade hídrica dos reservatórios, para o auxílio no manejo das terras cultivadas e na identificação da cobertura de vegetação nativa, e para o planejamento e a gestão ambiental, principalmente em casos onde haja necessidade de aplicação de técnicas de recuperação de áreas nativas degradadas, possibilitando também a delimitação de áreas de preservação ambiental. 
Ressalta-se que faz-se necessário em estudos futuros o emprego de estações meteorológicas, em áreas próximas a área de estudo, a fim de validar e confrontar os dados obtidos, considerando a atenuação decorrente da proximidade do espelho d'água existente, o qual pode estar influenciando no micro clima local.

\section{REFERÊNCIAS}

ALLEN, R.G.; TASUMI, M.; TREZZA, R. SEBAL (Surface Energy Balance Algorithms for Land): advanced training and user's manual - Idaho Implementation, version 1.0, 97 p., 2002.

ARAUJO, I.R. de; SILVA, H.P. da; LOPES, A. da S.; ALENCAR, B.P.B. de; SILVA, H.D.B. da. Cálculo de NDVI no suporte ao estudo de desertificação no município de Orocó - PE. In: SIMPÓSIO BRASILEIRO DE CIÊNCIAS GEODÉSICAS E TECNOLOGIAS DA GEOINFORMAÇÃO, 3., 2010, Recife. Anais... Recife, 2011. p. 01-06.

ARRAES, F.D.D.; ANDRADE, E.M. de; SILVA, B.B. da. Dinâmica do balanço de energia sobre o açude Orós e suas adjacências. Revista Caatinga, Mossoro, v. 25, n. 1, p. 119-127, jan.-mar., 2012.

BARBOSA, H. A.; HUETI, A. R.; BAETHGEN, W. E. A 20 year study of NDVI variability over the Northeast Region of Brazil. Journal of Arid Environments, v. 67, n. 2, p. 288-307, 2006.

BEZERRA, J. M.; MOURA, G. B. A.; SILVA, B. B.; LOPES, P. M. O.; SILVA, E. F. F. Parâmetros biofísicos obtidos por sensoriamento remoto em região semiárida do estado do Rio Grande do Norte, Brasil. Revista Brasileira de Engenharia Agrícola e Ambiental, v.18, n.1, p.73-84, 2014.

BEZERRA JÚNIOR, J.G.O.; SILVA, N.M. da. Caracterização geoambiental da microrregião do seridó oriental do Rio Grande do Norte. Holos, ano 23, v.2, 2007.

CHANDER, G.; MARKHAM, B. Revised Landsat-5 TM radiometric calibration procedures and post calibration dynamic ranges. IEEE Transactions on Geoscience and Remote Sensing, New York, v. 41, n. 11, p. 2674-2677. 2003.

CUNHA, J.E. de B.L.; RUFINO, I.A.A.; SILVA, B.B. da; CHAVES, I. de B. Dinâmica da cobertura vegetal para a Bacia de São João do Rio do Peixe, PB, utilizando-se sensoriamento remoto. Revista Brasileira de Engenharia Agrícola e Ambiental, v.16, p.539-548, 2012.

EMBRAPA. Levantamento Exploratório - Reconhecimento de solos do Estado do Rio Grande do Norte. Escala: 1:500.000. Embrapa - 1971. 
FECHINE, J.A.L.; GALVÍNCIO,D.J. Índice de vegetação por diferença normalizada das cidades de Salgueiro, Mirandiba, Carnaubeira da Penha e Floresta - localizadas no semiárido pernambucano. Revista Geográfica Acadêmica, v.2, n.3, p. 60-67, 2008

GARDA, E. C. Atlas do meio ambiente do Brasil. 2.ed. Brasília:

Terra Viva/EMBRAPA, 1996. 138p.

GURGEL, H. C.; FERREIRA, N. J.; LUIZ, A. J. B. Estudo da variabilidade do NDVI sobre o Brasil utilizando-se a análise de agrupamento. Revista Brasileira de Engenharia Agrícola e Ambiental, Campina Grande, v. 7, n. 1, p. 85-90, 2003.

OLIVEIRA, L.M.M. de.; MONTENEGRO, S.M.G.L.; ANTONINO, A.C.D.; SILVA, B.B. da.; MACHADO, C.C.C.; GALVÍNCIO, J.D. Análise quantitativa de parâmetros biofísicos de bacia hidrográfica obtidos por sensoriamento remoto. Pesquisa Agropecuária Brasileira, Brasília, v.47, n.9, p.1209-1217, set. 2012.

RIO GRANDE DO NORTE. INSTITUTO DE DESENVOLVIMENTO SUSTENTÁVEL E MEIO AMBIENTE DO RIO GRANDE DO NORTE. Perfil de seu município - Currais Novos. Natal, RN, v. 10, p. 1-23, 2008. Disponível em: < http://www.idema.rn.gov.br/contentproducao/aplicacao/idema/socio_economicos/arquivos/Pe rfil\%202008/Currais\%20Novos.pdf >. Acesso em: 21 jan. 2013.

SILVA, J.M.C.; TABARELLI, M.; FONSECA, M.T.; LINS, L.V. Biodiversidade da Caatinga: áreas e ações prioritárias para a conservação. Ministério do Meio Ambiente, Brasília. 2004.

SILVA, H.D.B. Estudo do Índice de Vegetação no Município de Floresta - PE, utilizando técnicas de Geoprocessamento e Sensoriamento Remoto. Universidade Federal de Rural de Pernambuco - UFRPE. Recife-PE, 2009.

SILVA, B.B. da; SILVA, S.T.A. da; GOMES, H.B. Alterações climáticas decorrentes de mudanças no uso da terra mediante sensoriamento remoto. Mercator, v.9, p.91-106, 2010.

SILVA, B.B. da; BRAGA, A.C.; BRAGA, C.C. Balanço de radiação no perímetro irrigado São Gonçalo - PB mediante imagens orbitais. Revista Caatinga, Mossoro, v. 24, n. 3, p. 145152, jul.-set., 2011.

\section{NOTAS DE AUTOR}

\section{CONTRIBUIÇÃO DE AUTORIA}

Hélio Nogueira Bezerra - Concepção. Coleta de dados, Análise de dados, Elaboração do manuscrito, revisão e aprovação da versão final do trabalho

Joel Medeiros Bezerra - Concepção e elaboração do manuscrito, conduzindo a validação dos dados e produção dos mapas temáticos. Participação ativa da discussão dos resultados; Revisão e aprovação da versão final do trabalho.

Caio Sérgio Pereira de Araújo -Revisão e aprovação da versão final do trabalho.

Arthur Mattos - Administração do projeto e Supervisão. Participação ativa da discussão dos resultados. 


\section{FINANCIAMENTO}

Os autores agradecem a Universidade Federal do Rio Grande do Norte (UFRN), e in memoria ao professor Dr. Arthur Matos, pelo incentivo a pesquisa acadêmica, a qual auxiliou o desenvolvimento e financiamento da pesquisa.

\section{LICENÇA DE USO}

Este artigo está licenciado sob a Licença Creative Commons CC-BY. Com essa licença você pode compartilhar, adaptar, criar para qualquer fim, desde que atribua a autoria da obra.

\section{HISTÓRICO}

Recebido em: 13-02-2017

Aprovado em: 19-06-2018 